\title{
Impact of clinical pharmacy services on renal transplant recipients' adherence and outcomes
}

\author{
Marie A Chisholm-Burns' \\ Christina A Spivey' \\ Charlene Garrett ${ }^{2}$ \\ Herbert McGinty ${ }^{2}$ \\ Laura L Mulloy ${ }^{3}$ \\ 'Department of Pharmacy Practice \\ and Science, The University of Arizona \\ College of Pharmacy, Tuscon, AZ, USA; \\ ${ }^{2}$ Medication Access Program (MAP), \\ University of Georgia College of \\ Pharmacy, Athens, GA, USA; ${ }^{3}$ Section \\ of Nephrology, Hypertension and \\ Transplantation Medicine, Medical \\ College of Georgia School of \\ Medicine, Augusta, GA, USA
}

\begin{abstract}
The purpose of this article is to provide a description of a clinical pharmacy services program implemented in a renal transplant clinic to improve medication access and adherence as well as health and economic outcomes among renal transplant recipients (RTRs). Following a team-based planning process and an informal survey of RTRs, a clinical pharmacy service intervention was implemented in the Medical College of Georgia renal transplant clinic. As part of the intervention, a clinical pharmacist reviewed and optimized medication therapy, provided instructions on how to take medication, and assisted with enrollment into medication assistance programs. Significant differences were found between RTRs who did and did not receive clinical pharmacy services on measures of adherence, health, economics, and quality of life. Clinical pharmacy services, as described in this article, have a positive impact on renal transplant recipients' medication adherence, health and economic outcomes, and health-related quality of life. The findings described here suggest that clinical pharmacy services are a viable and effective option for improving care for RTRs in an outpatient clinic setting.
\end{abstract}

Keywords: renal transplant recipients, immunosuppressant therapy adherence, health outcomes, economic outcomes

More than 179,000 kidney transplants have been performed in the United States since 1995 (UNOS 2008). In that same time period, more than 316,000 individuals were added to the kidney transplant waiting list, and greater than 42,000 individuals died while awaiting a kidney (renal) transplant. For those who receive renal transplants, maintenance of graft (transplanted kidney) function and improved health are among the most important post-transplant goals. Immunosuppressant therapy (IST) is integral to graft maintenance and survival, yet $20 \%$ to $70 \%$ of renal transplant recipients (RTRs) are nonadherent to their IST (improperly take their IST; Frazier et al 1994; Greenstein and Siegal 1998; Chisholm et al 2001; Butler et al 2004; Dew et al 2007). After waiting more than 3 years, incurring health care expenditures of over US $\$ 300,000$, and receiving a donated kidney for transplantation, it is a tragedy to lose graft function as the result of a preventable cause such as IST nonadherence. It is believed that more than one-third of graft failures are due to IST nonadherence (Gaston et al 1999). Butler and colleagues (2004) indicated that the odds of graft failure increased 7-fold in nonadherent RTRs compared to adherent RTRs, and concluded that significant improvement in graft survival could be expected from effective interventions to improve adherence. One such potential intervention is the implementation of clinical pharmacy services among RTRs as part of their post-transplant care.

Several studies have been conducted that demonstrate the value of pharmacy services in improving medication therapy adherence and health-related outcomes in various patient populations (Nola et al 2000; Till et al 2003; Kiel and McCord 2005; Paulós et al 2005; Lee et al 2006; McCord 2006; Armour et al 2007; Clark et al 2007; Murray et al 2007). For example, a pharmacy care program for elderly patients 
at risk for cardiovascular disease significantly improved medication adherence and blood pressure levels (Lee et al 2006). McCord (2006) found that drug therapy management and education services provided by a clinical pharmacist significantly improved clinical indicators such as hemoglobin $\mathrm{A}_{1 \mathrm{c}}$ levels among patients with diabetes. However, a review of the literature using PubMed, dates unlimited, produced very few studies of clinical pharmacy services as an intervention to improve medication adherence and/or outcomes among RTRs, indicating that there is a paucity of research concerning pharmacy services to improve IST adherence and RTRs' outcomes.

The value of pharmacist-provided care to transplant recipients has been recognized by the nation's foremost transplant organization, the United Network for Organ Sharing (UNOS). In order to qualify for UNOS membership, a transplant program should utilize at least one clinical pharmacist as part of the transplant team to provide pharmaceutical care to solid organ transplant recipients (UNOS 2007). It is our contention that clinical pharmacy services would be beneficial, not only as part of the care provided by the transplant team, but also in the outpatient transplant clinic setting. Thus, the purpose of this manuscript is to describe a successful program that utilizes clinical pharmacy services to improve medication adherence and outcomes of RTRs in an outpatient transplant clinic.

The Medical College of Georgia (MCG) Hospital and Clinics is a tertiary-care teaching hospital located in Augusta, Georgia. In late 1996, our team began exploring patient populations treated at MCG who could most benefit from clinical pharmacy services. We focused on those patients with chronic conditions who were required to take complicated, long-term medication regimens. The renal transplant population was immediately distinguished as a potential target for clinical pharmacy services for several reasons. MCG's renal transplant clinic provides care for more than 500 RTRs who must adhere to complex, expensive medication regimens, and as stated previously, the health-related consequences to RTRs of nonadherence to medication regimens can be dire (Chisholm 2006). As a result, RTRs are at increased risk for medication nonadherence, drug - drug interactions, and related adverse events. Additionally, immunosuppressant agents have influence on the cytochrome P450 system which also increases the possibility of drug interactions. According to anecdotal evidence, many patients served by the MCG renal transplant clinic were prone to medication nonadherence and/or suffered adverse drug events, and consequences of both included increased health care costs, negative health outcomes, and decreased quality of life. Our team hypothesized that clinical pharmacy services would increase medication adherence, reduce the number of adverse drug events, improve health outcomes, decrease health care costs, and increase quality of life among RTRs. We also believed that MCG's renal transplant clinic health care team, consisting of two nephrologists, nurses, and physician assistants, could benefit from the additional support provided by clinical pharmacy services. Therefore, we targeted RTRs and the MCG renal transplant clinic for clinical pharmacy services.

The first step in developing a clinical pharmacy program specifically for the MCG renal transplant clinic was a series of brainstorming sessions in which causes of adverse health outcomes related to medication (particularly barriers to IST adherence) were discussed. Aspects of the Health Decision Model (Eraker et al 1984), particularly factors affecting health decisions (in this case, the decision to adhere to IST) such as knowledge and sociodemographic factors, were considered during these sessions. A Fishbone diagram (Figure 1) was developed as a visual representation of what our team believed to be the major causes of nonadherence to medication regimens among RTRs. To assess the accuracy of those causes or barriers identified, we conducted an informal survey of RTRs at the renal transplant clinic. According to those surveyed, cost of IST and forgetfulness as well as other factors identified in Figure 1 were significant barriers to IST adherence (a study by Vasquez and colleagues [2003] further validated cost and forgetfulness as key barriers to medication adherence among RTRs). Based on these collective findings, a clinical pharmacy services intervention was developed, with the overarching goal of improving the care of MCG RTRs.

In February 1997, a randomized controlled trial of our clinical pharmacy services intervention was implemented in the MCG renal transplant clinic which addressed the barriers to IST adherence described in Figure 1 (Chisholm et al 2001). Adults at least 18 years of age, who received their first and only renal transplant at MCG between February 1997 and January 1999, were eligible to participate immediately following transplantation, and were enrolled into the study on a rolling basis (participants remained enrolled in the study for a 12-month period-the length of the intervention); the study was completed in January 2000. After consenting to participate in the study, RTRs were randomized into one of two groups, the intervention or the control. The RTRs in the intervention group $(n=12)$ received traditional care in combination with clinical pharmacy services. As part of the study protocol, on a monthly basis for the first 


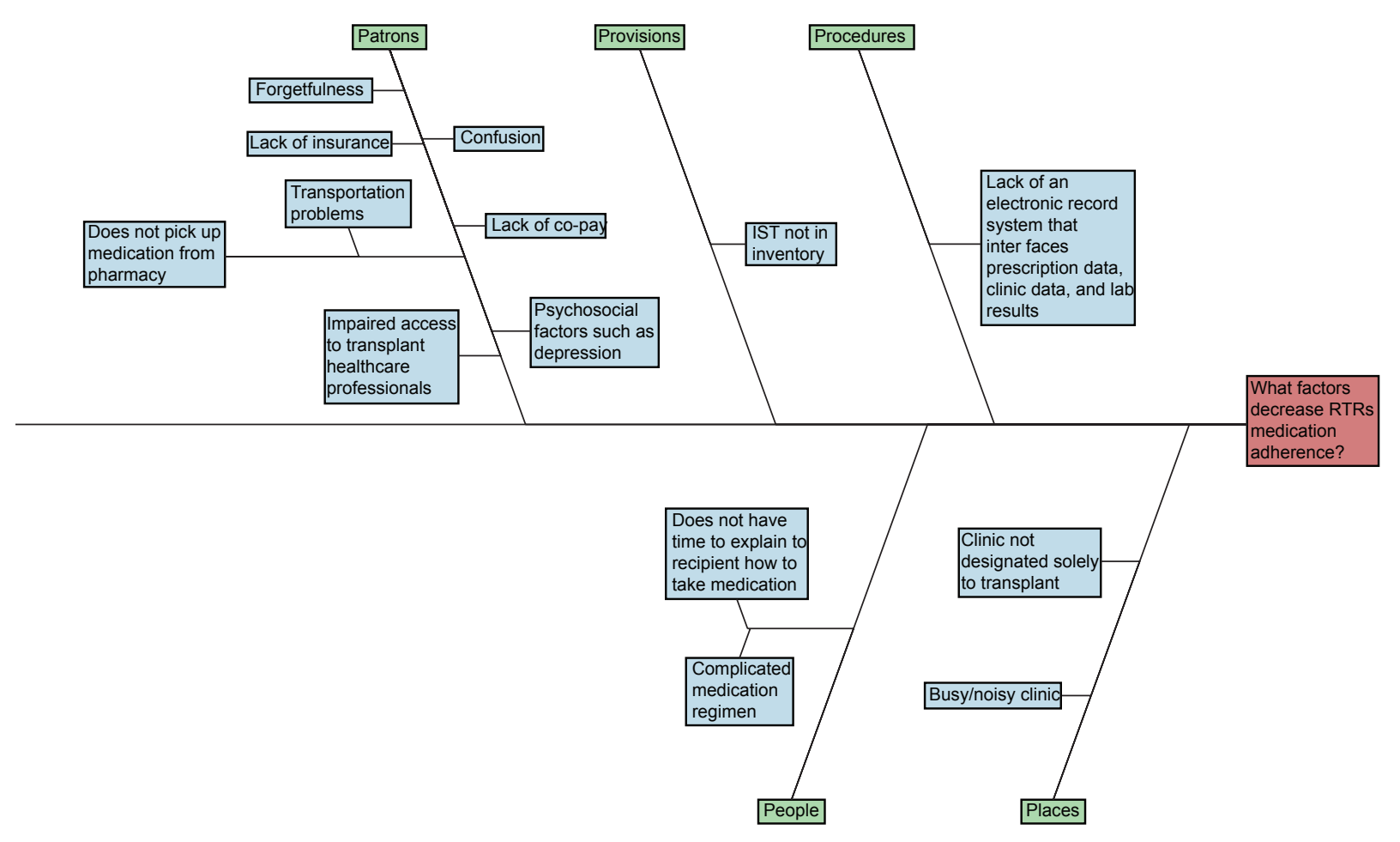

Figure I Fishbone diagram of possible causes of nonadherence.

Abbreviations: IST, immunosuppressant therapy; RTR, renal transplant recipient.

12 months post-transplant, a clinical pharmacist counseled RTRs assigned to the intervention group on their medication therapy, including providing instructions on how to properly take medications in order to promote medication adherence and health-related outcomes. Additional duties of the clinical pharmacist included: (1) reviewing RTRs' medication histories with emphasis on optimizing medication therapy and minimizing adverse events; (2) encouraging adherence to medications; (3) increasing access to medication by enrolling RTRs in pharmaceutical manufacturers' medication assistance programs; and (4) providing recommendations to the nephrologists, other health care team members, and RTRs to achieve desired health outcomes. RTRs assigned to the control group $(n=12)$ also received traditional care from the interdisciplinary clinic team (standard of care), but were not provided clinical pharmacy services.

The sample had a mean age of 42.9 years $(\mathrm{SD}=10.2)$ and was $75 \%$ male, $58 \%$ Caucasian, 38\% African American, and 4\% Hispanic (Chisholm et al 2001). The majority $(66.7 \%)$ received their kidney from a deceased donor, and approximately $88 \%$ were prescribed cyclosporine. At the end of the study, RTRs assigned to the intervention group had a higher mean adherence rate compared to those in the control group $(96.1 \% \pm 4.7$ vs $81.6 \% \pm 11.5 ; p<0.001$;
Chisholm et al 2001). RTRs in the intervention group also remained adherent for a longer period of time during the 12-month intervention compared to those RTRs assigned to the control group (a mean of 11 months $v s$. a mean of 9 months to first nonadherent month $[p<0.05]$; nonadherence was defined as an adherence rate of less than $80 \%$; Chisholm et al 2001). It is important to note that although our study sample was too small to detect graft rejection and/or failure, other studies provide evidence of a significant association between medication nonadherence and rejection/ failure (Vasquez et al 2003; Chisholm et al 2007). Based on this evidence, we speculate that the improvements in adherence demonstrated by RTRs as a result of clinical pharmacy services may play a role in long-term graft maintenance and survival.

The clinical pharmacist's efforts to eliminate cost as a barrier to adherence by enrolling RTRs in pharmaceutical manufacturers' medication assistance programs was considered a particularly successful outcome of the clinical pharmacy services. As part of the evaluation of our clinical pharmacy services intervention, we studied the cost-avoidance of enrolling qualified patients into manufacturers' immunosuppressant medication assistance programs (Chisholm et al 2000a). 
The clinical pharmacist enrolled RTRs into assistance programs for immunosuppressant medications from January 1, 1998 through December 31, 1998 if they met the following criteria: (1) needed financial assistance to purchase their immunosuppressant medications, and (2) qualified financially for the manufacturers' assistance programs. Sixty-one RTRs were enrolled in pharmaceutical manufacturers' assistance programs and a net cost avoidance of $\$ 69,233$ for the year 1998 was calculated with a benefit-to-cost ratio of approximately $4: 1$.

Supplemental randomized controlled trials examining this clinical pharmacy service program's quality and impact of on RTRs' health-related and economic outcomes have also been conducted. Compared to RTRs who did not receive clinical pharmacy services, RTRs who did receive clinical pharmacy services in the MCG renal transplant clinic (Table 1): (1) were more satisfied with the quality of their health care (Chisholm et al 1999a); (2) experienced fewer adverse drug reactions (Chisholm et al 2000b); (3) experienced improvements in their blood pressure and blood glucose levels (Chisholm et al 1999b, 1999c, 2002); and (4) had lower mean total health care costs per RTR (Vollenweider et al 2001). Additionally, the clinical pharmacist provided physicians with 844 pharmacotherapeutic recommendations during the program's initial (pilot) implementation period (Chisholm et al 2000c). Greater than $80 \%$ of the recommendations involved untreated indications, overdosage, subtherapeutic dosage, or medication use without an indication (Figure 2). Based upon the cumulative positive results of these studies, clinical pharmacy services are ongoing at the MCG renal transplant clinic.

In conclusion, clinical pharmacy services resulted in significant improvements in RTRs' clinical and economic outcomes. Although UNOS supports the use of a clinical pharmacist as a member of the transplant team and clinical pharmacy services have had positive effects in the management of other chronic disease states, little evidence from randomized controlled trials and other studies of clinical pharmacy services to improve post-transplant care for RTRs was found in the literature. We recognize the challenges involved in implementing clinical pharmacy services among transplant recipients including lack of knowledge/experience in designing and administering such services as well as difficulty in procuring funding and reimbursement for services. Yet, the success of our program demonstrates that clinical pharmacy services are feasible within an outpatient transplant clinic and have a beneficial impact on outcomes among transplant recipients. Thus, this article addresses a strategy for effective implementation of clinical pharmacy services and may provide a template for use in other

Table I Impact of clinical pharmacy services at MCG renal transplant clinic on renal transplant recipients' outcomes

\begin{tabular}{|c|c|c|c|c|}
\hline Reference & $\begin{array}{l}\text { Study } \\
\text { design }\end{array}$ & Number of RTRs & Outcome(s) examined & Results \\
\hline Chisholm et al I999b & $\mathrm{RCT}$ & I8 (intervention); I5 (control) & Blood pressure levels & $\begin{array}{l}\text { Significant SBP and DBP level } \\
\text { improvement in intervention } \\
\text { group }(p<0.05)\end{array}$ \\
\hline Chisholm et al I999c & $\mathrm{RCT}$ & I8 (intervention); I5 (control) & Blood glucose levels & $\begin{array}{l}\text { Significant fasting blood } \\
\text { glucose level improvement in } \\
\text { intervention group }(p<0.05)\end{array}$ \\
\hline Chisholm et al 1999a & $\mathrm{RCT}$ & 2I (intervention); I7 (control) & $\begin{array}{l}\text { Satisfaction with health } \\
\text { care quality }\end{array}$ & $\begin{array}{l}\text { Intervention group more } \\
\text { satisfied with health care } \\
\text { Quality }(p<0.05)\end{array}$ \\
\hline Chisholm et al $2000 \mathrm{~b}$ & $\mathrm{RCT}$ & 28 (intervention); 26 (control) & Adverse drug reactions & $\begin{array}{l}\text { Significantly fewer ADRs in } \\
\text { intervention group }(p<0.05)\end{array}$ \\
\hline Vollenweider et al 200I & $\mathrm{RCT}$ & 26 (intervention); 28 (control) & Health care costs & $\begin{array}{l}\text { Intervention group had mean } \\
\text { total costs of } \$ 2614 \text { less } \\
\text { per RTR than control group; } \\
\text { clinical pharmacy services } \\
\text { results in cost savings of } \\
\$ 192,757\end{array}$ \\
\hline Chisholm et al 2002 & $\mathrm{RCT}$ & I3 (intervention); I0 (control) & $\begin{array}{l}\text { Blood pressure } \\
\text { of African-American RTRs }\end{array}$ & $\begin{array}{l}\text { Significant SBP and DBP level } \\
\text { improvement in intervention } \\
\text { group }(p<0.05)\end{array}$ \\
\hline
\end{tabular}

Abbreviations: ADR, adverse drug reaction; DBP, diastolic blood pressure; MCG, Medical College of Georgia; RCT, randomized controlled trial; RTR, renal transplant recipient; SBP, systolic blood pressure. 


\section{Pareto chart of type of problem}

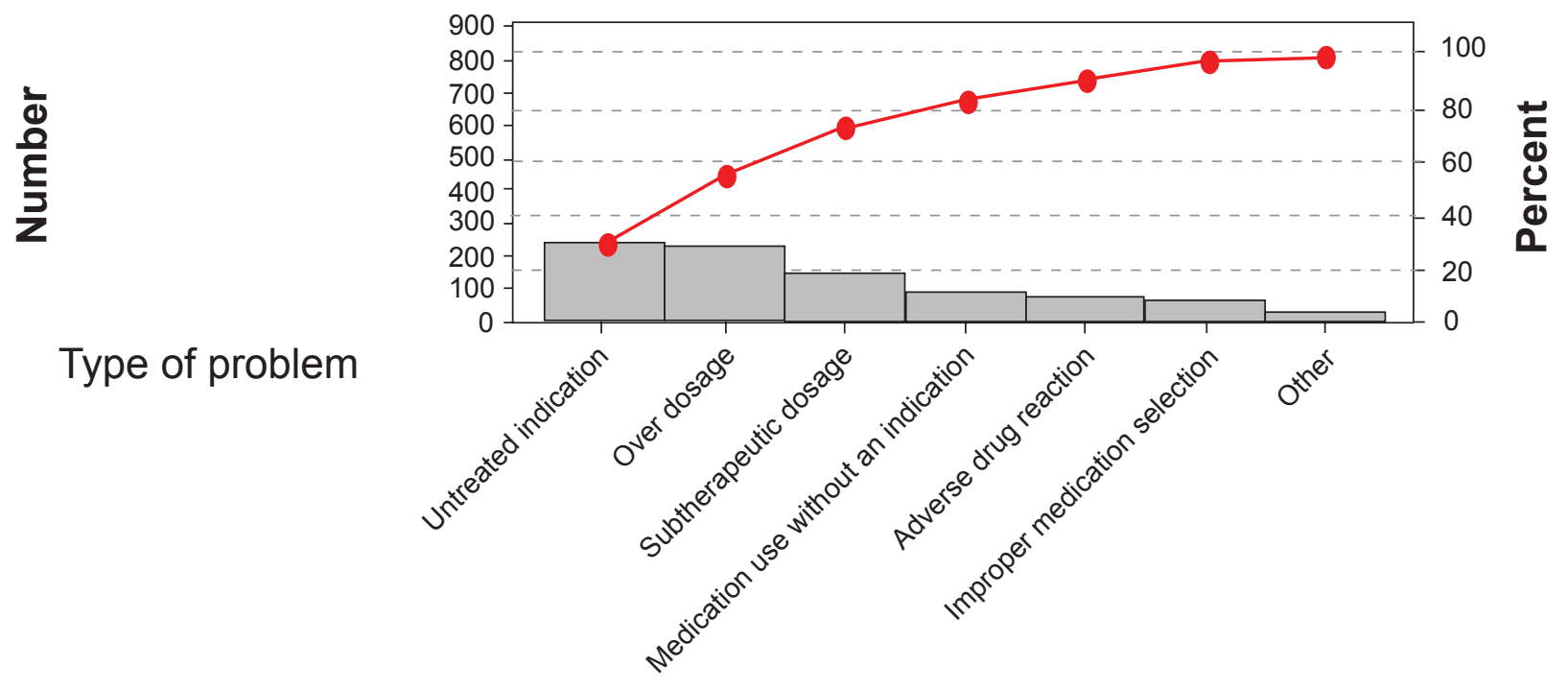

Figure 2 Pareto chart of problems addressed by clinical pharmacist's recommendations (Chisholm et al 2000c).

outpatient transplant care settings. Future studies should focus on implementing clinical pharmacy services in other transplant clinics, evaluating modifications to the clinical pharmacy program that will promote cost-effectiveness while maintaining positive adherence and health outcomes (such as reducing the time period for provision of clinical pharmacy services), and assessing the long-term impact of clinical pharmacy services in the transplant population.

\section{Disclosure}

The authors report no conflicts of interest in this work.

\section{References}

Armour C, Bosnic-Anticevich S, Brillant M, et al. 2007. Pharmacy Asthma Care Program (PACP) improves outcomes for patients in the community. Thorax, 62:496-502.

Butler JA, Roderick P, Mullee M, et al. 2004. Frequency and impact of nonadherence to immunosuppressants after renal transplantation: a systematic review. Transplantation, 77:769-76.

Chisholm MA, Kwong WJ, Spivey CA. 2007. Associations of characteristics of renal transplant recipients with clinicians' perceptions of adherence to immunosuppressant therapy. Transplantation, $84: 1145-50$.

Chisholm MA. 2006. A renal transplantation advanced pharmacy practice experience. Am J Pharm Educ, 70:3.

Chisholm MA, Mulloy LL, Jagadeesan M, et al. 2002. Effect of clinical pharmacy services on the blood pressure of African-American renal transplant patients. Ethn Dis, 12:392-7.

Chisholm MA, Mulloy LL, Jagadeesan M, et al. 2001. Impact of clinical pharmacy services on renal transplant patients' compliance with immunosuppressive medications. Clin Transplant, 15:330-6.

Chisholm MA, Reinhardt BO, Vollenweider LJ, et al. 1999a. Renal transplant clinic patients' satisfaction with health care quality: effect of pharmaceutical care services. Pharmacotherapy, 19:1228.
Chisholm MA, Vollenweider LJ, Reinhardt BO, et al. 1999b. Effect of pharmaceutical care services on the blood pressure of renal transplant patients. Pharmacotherapy, 19:1222.

Chisholm MA, Reinhardt BO, Vollenweider LJ, et al. 1999c. Effect of pharmaceutical care services on renal transplant patients' blood glucose levels. International Pharmaceutical Abstracts, $36: 2180-1$.

Chisholm MA, Vollenweider LJ, Mulloy LL, et al. 2000a. Cost-benefit analysis of a clinical pharmacist-managed medication assistance program in a renal transplant clinic. Clin Transplant, 14:304-7.

Chisholm MA, Vollenweider LJ, Mulloy LL, et al. 2000b. Pharmaceutical care services reduce adverse drug reactions in renal transplant patients. Pharmacotherapy, 20:1270.

Chisholm MA, Vollenweider LJ, Mulloy LL, et al. 2000c. Direct patient care services provided by a pharmacist on a multidisciplinary renal transplant team. Am J Health Syst Pharm, 57:1994-6.

Clark PM, Karagoz T, Apikoglu-Rabus S, et al. 2007. Effect of pharmacist-led patient education on adherence to tuberculosis treatment. Am J Health Syst Pharm, 64:497-505.

Dew MA, DiMartini AF, De Vito Dabbs, A, et al. 2007. Rates and risk factors for nonadherence to the medical regimen after adult solid organ transplantation. Transplantation, 83:858-73.

Eraker SA, Kirscht JP, Becker MH. 1984. Understanding and improving patient compliance. Ann Intern Med, 100:258-68.

Frazier P, Davis-Ali S, Dahl K. 1994. Correlates of noncompliance among renal transplant recipients. Clin Transplant, 8:550-7.

Gaston R, Hudson S, War M, et al. 1999. Late renal allograft loss: noncompliance masquerading as chronic rejection. Transplant Proc, $31: 21 \mathrm{~s}-23 \mathrm{~s}$.

Greenstein S, Siegal B. 1998. Compliance and noncompliance in patients with functioning renal transplant: a multicenter study. Transplantation, 66:1718-26.

Kiel PJ, McCord AD. 2005. Pharmacist impact on clinical outcomes in a diabetes disease management program via collaborative practice. Ann Pharmacother, 39:1828-32.

Lee JK, Grace KA, Taylor AJ. 2006. Effect of a pharmacy care program on medication adherence and persistence, blood pressure, and low-density lipoprotein cholesterol: a randomized controlled trial. JAMA, 296:2563-71. 
McCord AD. 2006. Clinical impact of a pharmacist-managed diabetes mellitus drug therapy management service. Pharmacotherapy, 26:248-53.

Murray MD, Young J, Hoke S, et al. 2007. Pharmacist intervention to improve medication adherence in heart failure: a randomized trial. Ann Intern Med, 146:714-25.

Nola KM, Gourley DR, Portner TS, et al. 2000. Clinical and humanistic outcomes of a lipid management program in the community pharmacy setting. J Am Pharm Assoc, 40:166-73.

Paulós CP, Nygren CE, Celedón C, et al. 2005. Impact of a pharmaceutical care program in a community pharmacy on patients with dyslipidemia. Ann Pharmacother, 39:939-43.

Till LT, Voris JC, Horst JB. 2003. Assessment of clinical pharmacist management of lipid-lowering therapy in a primary care setting. J Manage Care Pharm, 9:269-73.
[UNOS] United Network for Organ Sharing. 2007. Attachment I to Appendix B of UNOS ByLaws: Designated Transplant Program Criteria [online]. Accessed on Jan 15, 2008. URL: http://www. unos.org/policiesandBylaws2/bylaws/UNOSByLaws/pdfs/ bylaw_116.pdf.

[UNOS] United Network for Organ Sharing. 2008. Transplants in the US by recipient gender [online]. Accessed on Jan 8, 2008. URL: http://www. optn.org/latestData/rptData.asp.

Vasquez EM, Tanzi M, Benedetti E, et al. 2003. Medication noncompliance after kidney transplantation. Am J Health Syst Pharm, 60:266-9.

Vollenweider LJ, DiPiro JT, Chisholm MA. 2001. Economic evaluation of direct patient care pharmacy services to renal transplant clinic patients. Am J Transplant, 1(Suppl 1):273. 